\title{
Magnetic Structure and Magnetic Properties of Synthetic Lindgrenite, $\mathrm{Cu}_{3}(\mathrm{OH})_{2}\left(\mathrm{MoO}_{4}\right)_{2}$
}

\section{Serge Vilminot, ${ }^{*} \dagger$ Gilles André,‡ Mireille Richard-Plouet,§ Françoise Bourée-Vigneron, ${ }^{\ddagger}$ and Mohamedally Kurmoo*,\#}

Groupe des Matériaux Inorganiques, IPCMS UMR CNRS-ULP 7504, 23 rue du Loess, BP 43, 67034 Strasbourg Cedex 2, France, Laboratoire Léon Brillouin, CEA-CNRS, CEA Saclay, 91191 Gif-sur-Yvette Cedex, France, Institut des Matériaux Jean Rouxel, Laboratoire de Chimie des Solides, 2 rue de la Houssinière, BP 32229, 44322 Nantes Cedex, France, Laboratoire de Chimie de Coordination Organique, CNRS-UMR7140, Tectonique Moléculaire du Solide, Université Louis Pasteur, Institut Le Bel, 4 rue Blaise Pascal, 67000 Strasbourg Cedex 01, France

\section{Supporting Information.}

Table S1. Energies of the Infrared Vibration Bands $\left(\mathrm{cm}^{-1}\right)$ and their Assignments

\begin{tabular}{|c|c|c|}
\hline 1 & 2 & Assignment \\
\hline $3402(w)$ & & O-H symmetric stretching \\
\hline $3338(\mathrm{~m})$ & & $\mathrm{O}-\mathrm{H}$ antisymmetric stretching \\
\hline \multicolumn{3}{|l|}{$3258(w)$} \\
\hline & $2554(\mathrm{vw})$ & O-D symmetric stretching \\
\hline & $2488(\mathrm{~s})$ & O-D antisymmetric stretching \\
\hline & 2410 (vw br) & \\
\hline 958 (vs) & 952 (vs) & $v_{1} \mathrm{MoO}_{4}$ \\
\hline 914 (vs) & $911(\mathrm{~s})$ & $v_{3} \mathrm{MoO}_{4}$ \\
\hline 868 (vs) & 865 (vs) & $v_{3} \mathrm{MoO}_{4}$ \\
\hline \multirow[t]{3}{*}{825 (vs, broad) } & 831(vs, broad) & $v_{3} \mathrm{MoO}_{4}$ \\
\hline & $671(s)$ & O-D libration \\
\hline & $635(s)$ & O-D libration \\
\hline $455(\mathrm{~s})$ & $440(\mathrm{~m})$ & $\mathrm{Cu}-\mathrm{OH}(\mathrm{D})$ stretching \\
\hline $420(s)$ & $410(w)$ & $\mathrm{OH}$ \\
\hline $387(\mathrm{~m})$ & $388(\mathrm{~m})$ & $v_{2}$ and $v_{4} \mathrm{MoO}_{4}$ \\
\hline $352(\mathrm{~m})$ & $349(\mathrm{~m})$ & $v_{2}$ and $v_{4} \mathrm{MoO}_{4}$ \\
\hline $341(\mathrm{~m})$ & $341(\mathrm{~m})$ & $v_{2}$ and $v_{4} \mathrm{MoO}_{4}$ \\
\hline
\end{tabular}

Table S2. Interatomic Distances $(\AA)$ and Angles $\left({ }^{\circ}\right)$ from the Neutron Data at $300 \mathrm{~K}$.

$\begin{array}{ll}\mathrm{Cu} 1-\mathrm{O} 2 & 2.409(3) \times 2 \\ \mathrm{Cu} 1-\mathrm{O} 4 & 1.953(3) \times 2 \\ \mathrm{Cu} 1-\mathrm{OD} & 2.016(3) \times 2 \\ <\mathrm{Cu} 1-\mathrm{O}> & 2.126\end{array}$

$\begin{array}{ll}\text { O2-Cu1-O2 } & 180.0 \\ \text { O2-Cu1-O4 } & 90.2(2) \times 2 \\ \text { O2-Cu1-O4 } & 89.8(2) \times 2 \\ \text { O2-Cu1-OD } & 81.3(2) \times 2 \\ \text { O2-Cu1-OD } & 98.7(2) \times 2 \\ \text { O4-Cu1-O4 } & 180.0 \\ \text { O4-Cu1-OD } & 93.0(2) \times 2\end{array}$




$\begin{array}{ll}\mathrm{Cu} 2-\mathrm{O} 1 & 1.928(4) \\ \mathrm{Cu} 2-\mathrm{O} 2 & 2.306(3) \\ \mathrm{Cu} 2-\mathrm{O} 3 & 1.934(4) \\ \mathrm{Cu} 2-\mathrm{O} 4 & 2.476(3) \\ \mathrm{Cu} 2-\mathrm{OD} & 1.986(4) \\ \mathrm{Cu} 2-\mathrm{OD} & 1.999(4) \\ <\mathrm{Cu} 2-\mathrm{O}> & 2.105\end{array}$
Mo-O1 1.760(4)
Mo-O2 1.754(4)
Mo-O3 1.772(4)
Mo-O4 1.774(4)
<Mo-O> $\quad 1.765$

OD-D $\quad 0.956(4)$

D...O3 1.966(4)

\section{$\underline{\mathrm{Cu}-\mathrm{O}-\mathrm{Cu} \text { angles }}$}

$\begin{array}{lll}\mathrm{Cu} 1-\mathrm{O} 2-\mathrm{Cu} 2 & 86.2(1) & 85.6(3)^{*} \\ \mathrm{Cu} 1-\mathrm{OH}-\mathrm{Cu} 2 & 107.2(2) & 105.0(5)^{*} \\ \mathrm{Cu} 1-\mathrm{O} 4-\mathrm{Cu} 2 & 89.9(1) & 91.4(4)^{*} \\ \mathrm{Cu} 1-\mathrm{OH}-\mathrm{Cu} 2 & 103.4(2) & 103.3(4)^{*} \\ \mathrm{Cu} 2-\mathrm{OH}-\mathrm{Cu} 2 & 98.5(2) \times 2 & 95.5(5)^{*} \times 2\end{array}$

*Values are those at $1.5 \mathrm{~K}$
O4-Cu1-OD $\quad 87.0(2) \times 2$

OH-Cu1-OD $\quad 180.0$

$\mathrm{O} 1-\mathrm{Cu} 2-\mathrm{O} 2 \quad 90.3(2)$

$\mathrm{O} 1-\mathrm{Cu} 2-\mathrm{O} 3 \quad 96.3(3)$

$\mathrm{O} 1-\mathrm{Cu} 2-\mathrm{O} 4 \quad 81.5(2)$

O1-Cu2-OD 95.3(2)

$\mathrm{O} 1-\mathrm{Cu} 2-\mathrm{OD} \quad 155.6(3)$

$\mathrm{O} 2-\mathrm{Cu} 2-\mathrm{O} 3 \quad 87.9(2)$

$\mathrm{O} 2-\mathrm{Cu} 2-\mathrm{O} 4 \quad 170.3(2)$

$\mathrm{O} 2-\mathrm{Cu} 2-\mathrm{OD} \quad$ 84.6(2)

$\mathrm{O} 2-\mathrm{Cu} 2-\mathrm{OD} \quad 113.3(2)$

$\mathrm{O} 3-\mathrm{Cu} 2-\mathrm{O} 4 \quad 98.1(2)$

O3-Cu2-OD 166.2(3)

O3-Cu2-OD 90.9(2)

O4-Cu2-OD 91.0(2)

O4-Cu2-OD 74.4(2)

OD-Cu2-OD 81.5(2)

O1-Mo-O2 108.4(3)

O1-Mo-O3 110.3(3)

O1-Mo-O4 110.8(3)

O2-Mo-O3 108.2(3)

O2-Mo-O4 108.6(3)

O3-Mo-O4 110.4(3)

OD-D...O3 150.3(5)

Table S3. Moments and Reliability Factors Obtained in the Neutron Data Refinements.

\begin{tabular}{lll}
\hline $\mathbf{T}(\mathbf{K})$ & $\mathbf{M}_{\mathbf{x}}\left(\boldsymbol{\mu}_{\mathbf{B}}\right)$ & $\mathbf{R}_{\text {magnetic }}(\boldsymbol{\%})$ \\
\hline 1.5 & $0.77(4)$ & 4.9 \\
4.0 & $0.78(4)$ & 4.7 \\
5.9 & $0.77(4)$ & 4.4 \\
7.9 & $0.72(4)$ & 5.3 \\
9.8 & $0.68(5)$ & 4.2 \\
10.8 & $0.57(5)$ & 4.9 \\
11.8 & $0.30(8)$ & 5.2 \\
12.7 & 0 & \\
13.7 & 0 & \\
17.8 & 0 &
\end{tabular}




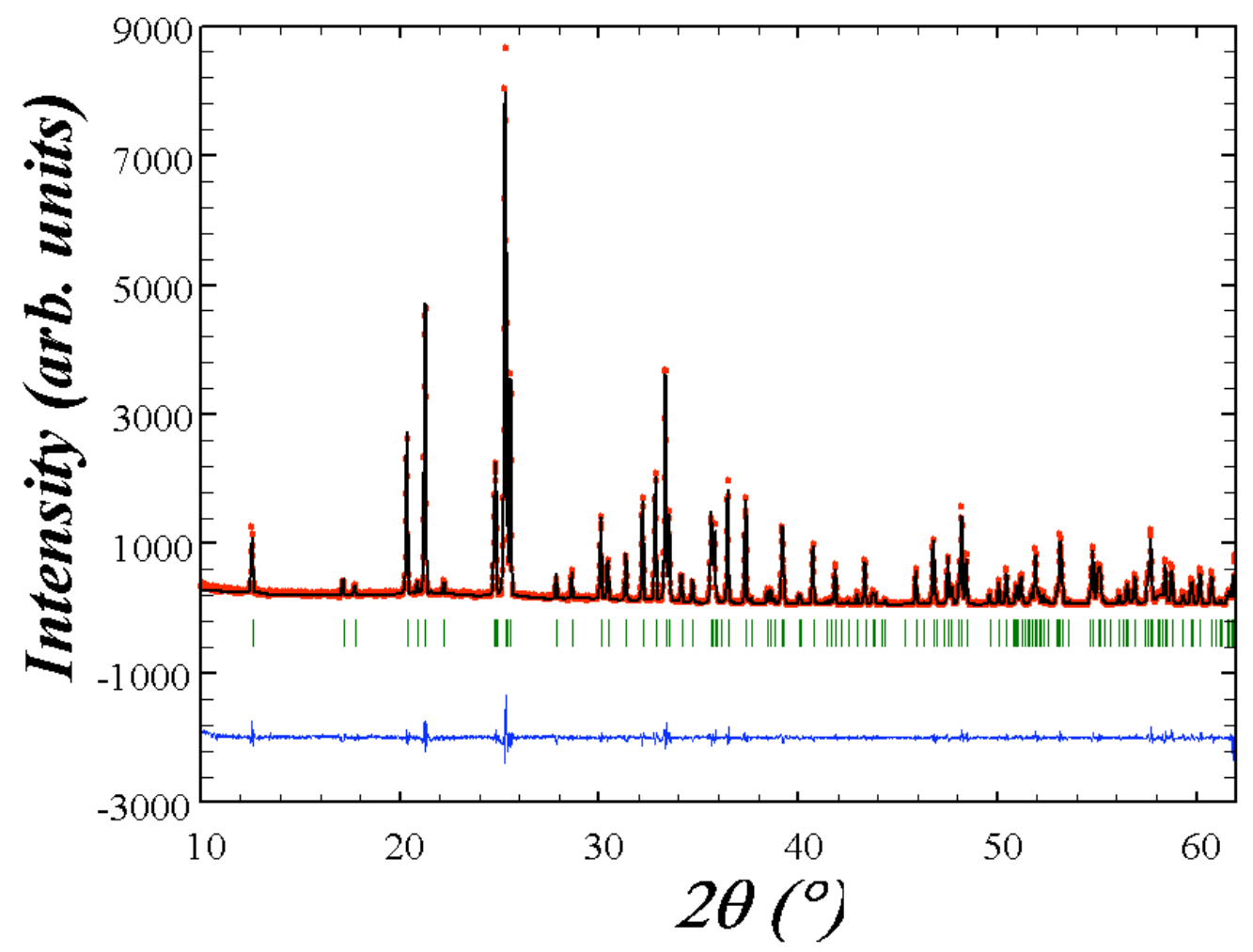

Figure S1. Observed (red circles) and calculated (black line) profiles of the X-ray powder diffraction of $\mathrm{Cu}_{3}(\mathrm{OH})_{2}\left(\mathrm{MoO}_{4}\right)_{2}$ obtained on a Siemens D5000 diffractometer with monochromatized $\mathrm{CuK \alpha} \alpha_{1}$ radiation $(\lambda=1.5406 \AA)$ at $300 \mathrm{~K}$ with position of the Bragg reflections (short vertical green lines) and difference between observed and calculated profiles (blue).

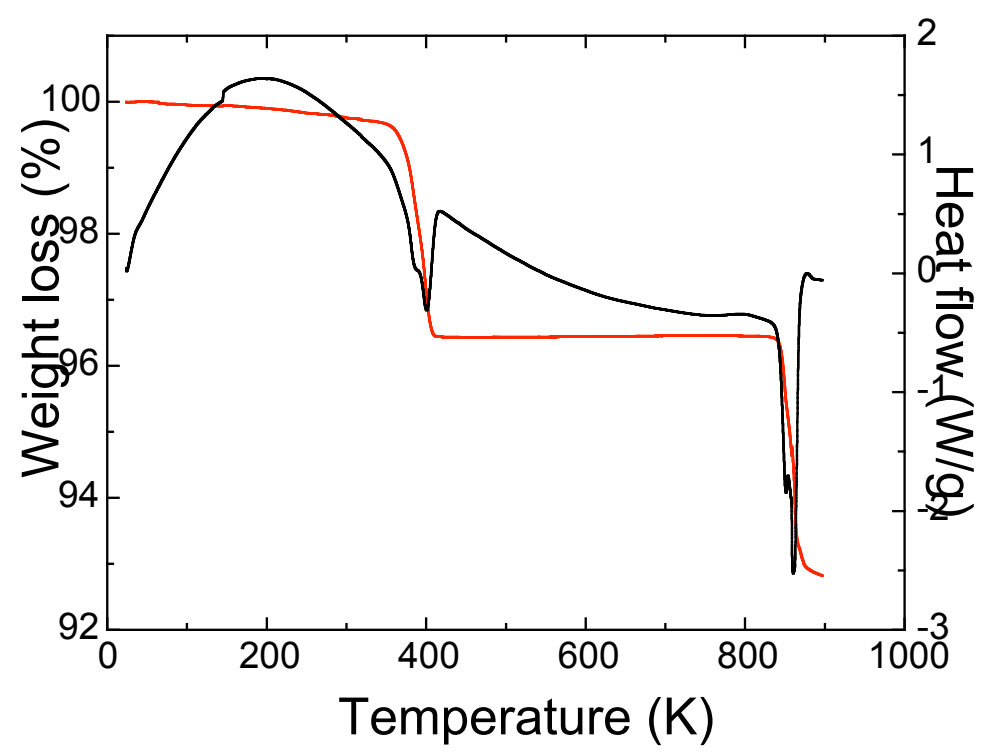

Figure S2. TG-DTA trace of $\mathrm{Cu}_{3}(\mathrm{OH})_{2}\left(\mathrm{MoO}_{4}\right)_{2}$ under air at $5^{\circ} \mathrm{C} / \mathrm{min}$ heating rate. 


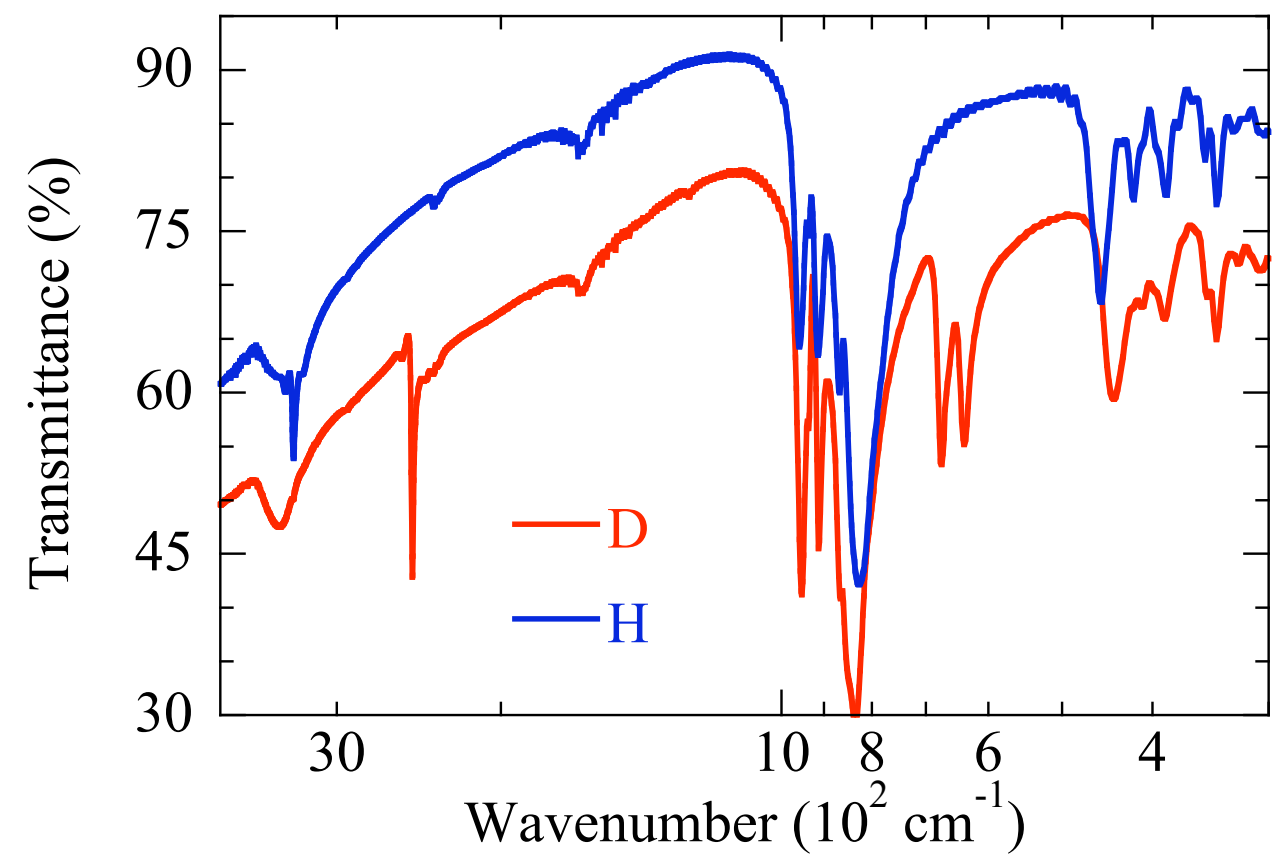

Figure S3. IR spectra of $\mathrm{Cu}_{3}(\mathrm{OH})_{2}\left(\mathrm{MoO}_{4}\right)_{2}$ and $\mathrm{Cu}_{3}(\mathrm{OD})_{2}\left(\mathrm{MoO}_{4}\right)_{2}$. 


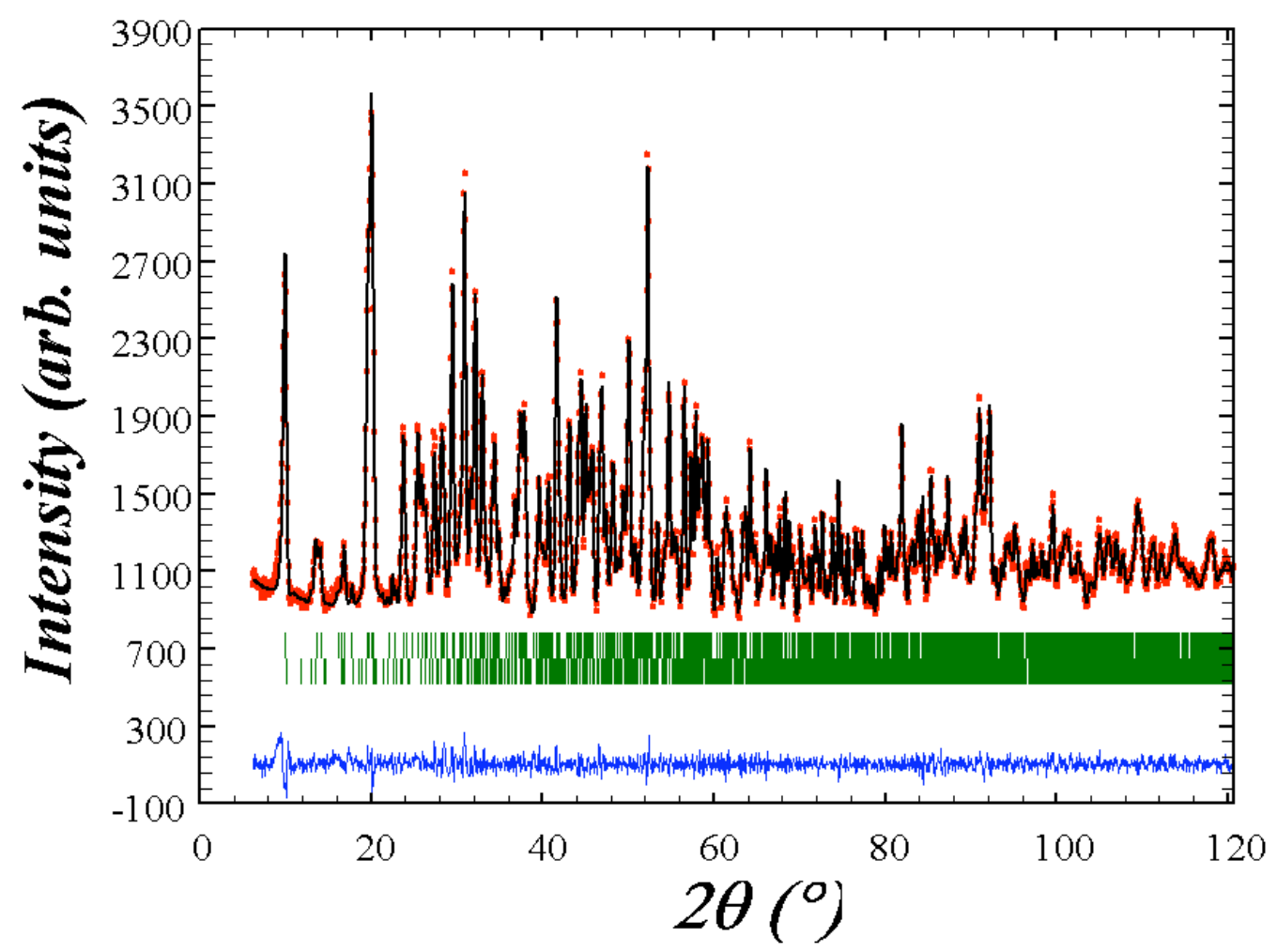

Figure S4. Observed (red circles) and calculated (black line) profiles of the neutron powder diffraction pattern of $\mathrm{Cu}_{3}(\mathrm{OD})_{2}\left(\mathrm{MoO}_{4}\right)_{2}$ obtained on the 3T2 diffractometer $(\lambda=1.2244 \AA)$ at $300 \mathrm{~K}$ with position of the Bragg reflections of $\mathrm{Cu}_{3}(\mathrm{OD})_{2}\left(\mathrm{MoO}_{4}\right)_{2}$ (upper short vertical green lines) and of $\mathrm{Na}_{2} \mathrm{MoO}_{4} \cdot 2 \mathrm{D}_{2} \mathrm{O}$ (lower short vertical green lines) and difference between observed and calculated profiles (blue).

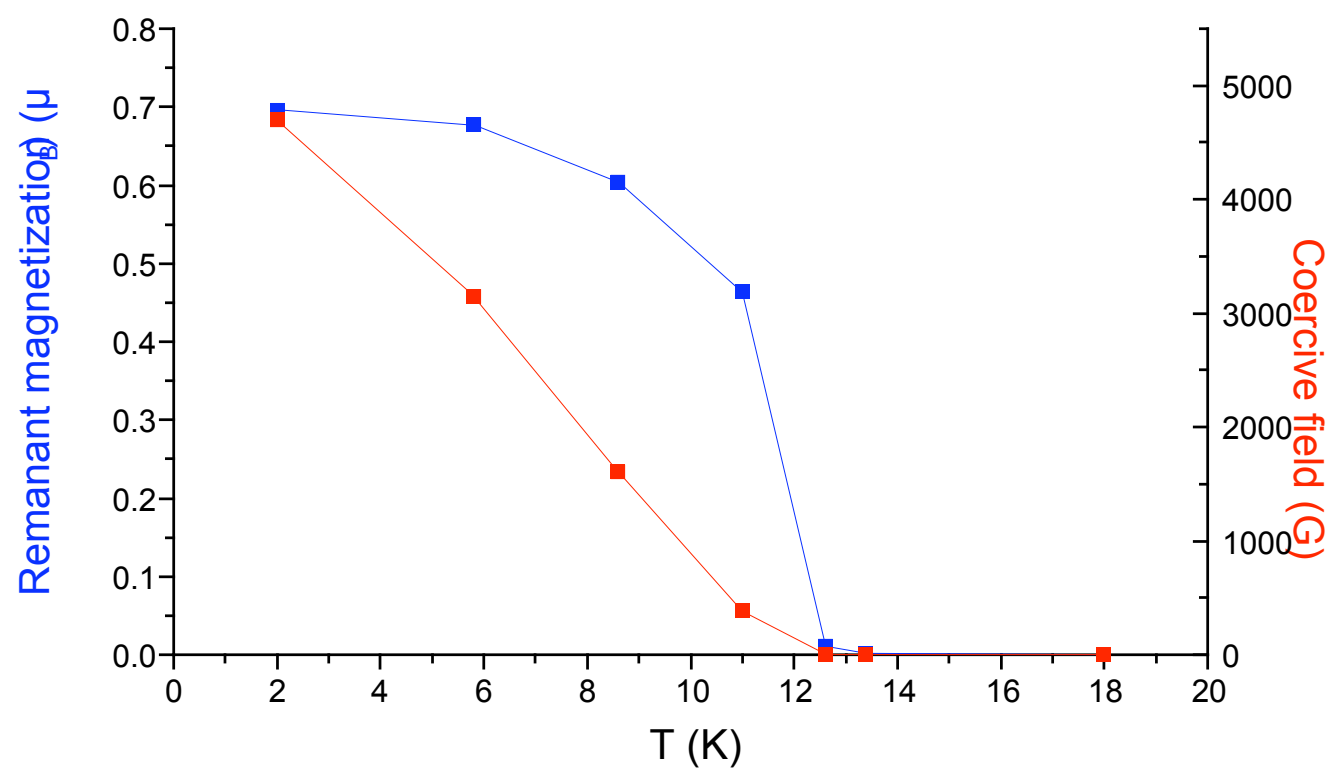

Figure S5. Evolution of the remanant magnetization and coercive field with temperature. 


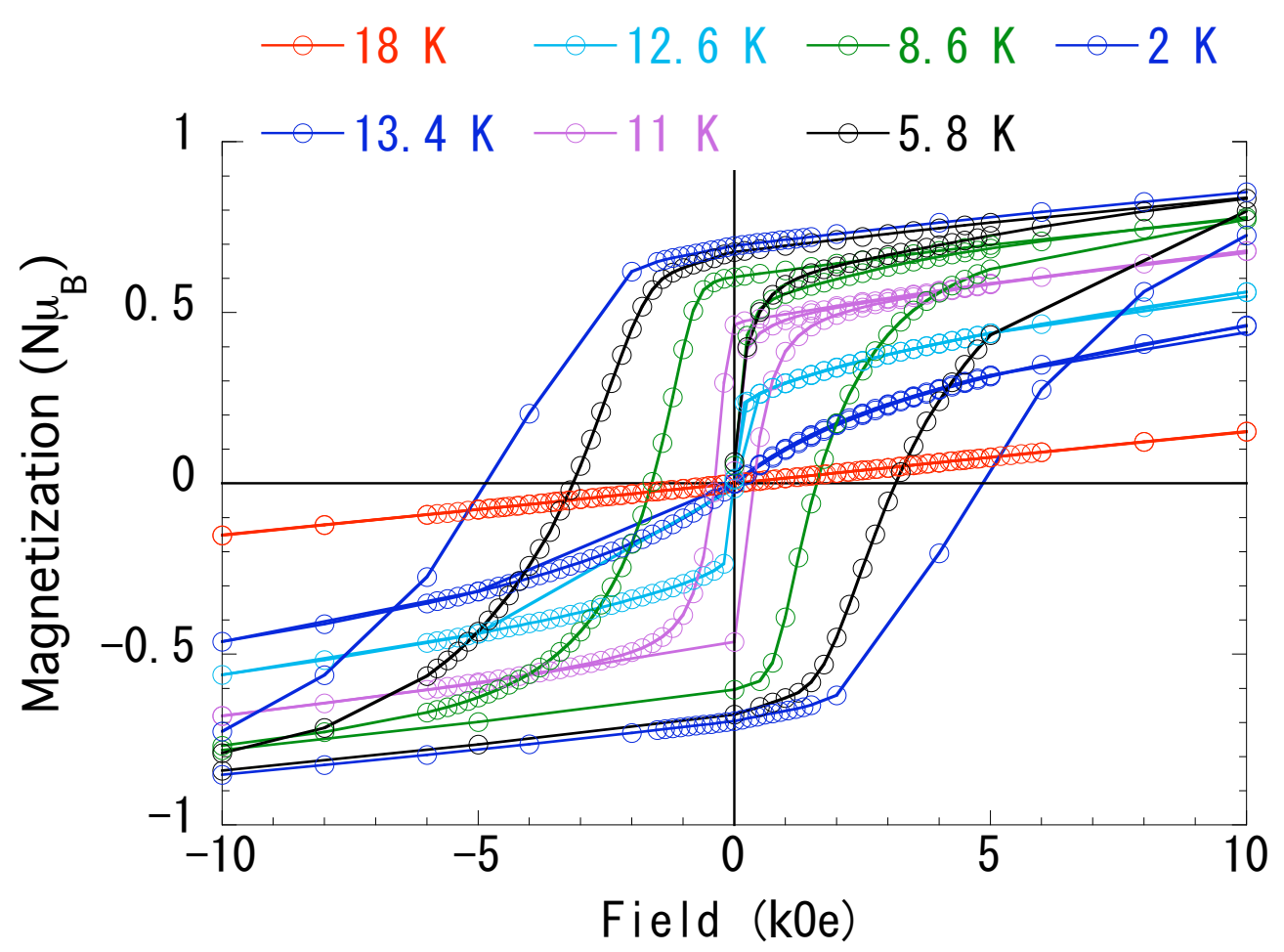

Figure S6. Isothermal magnetization at different temperatures.

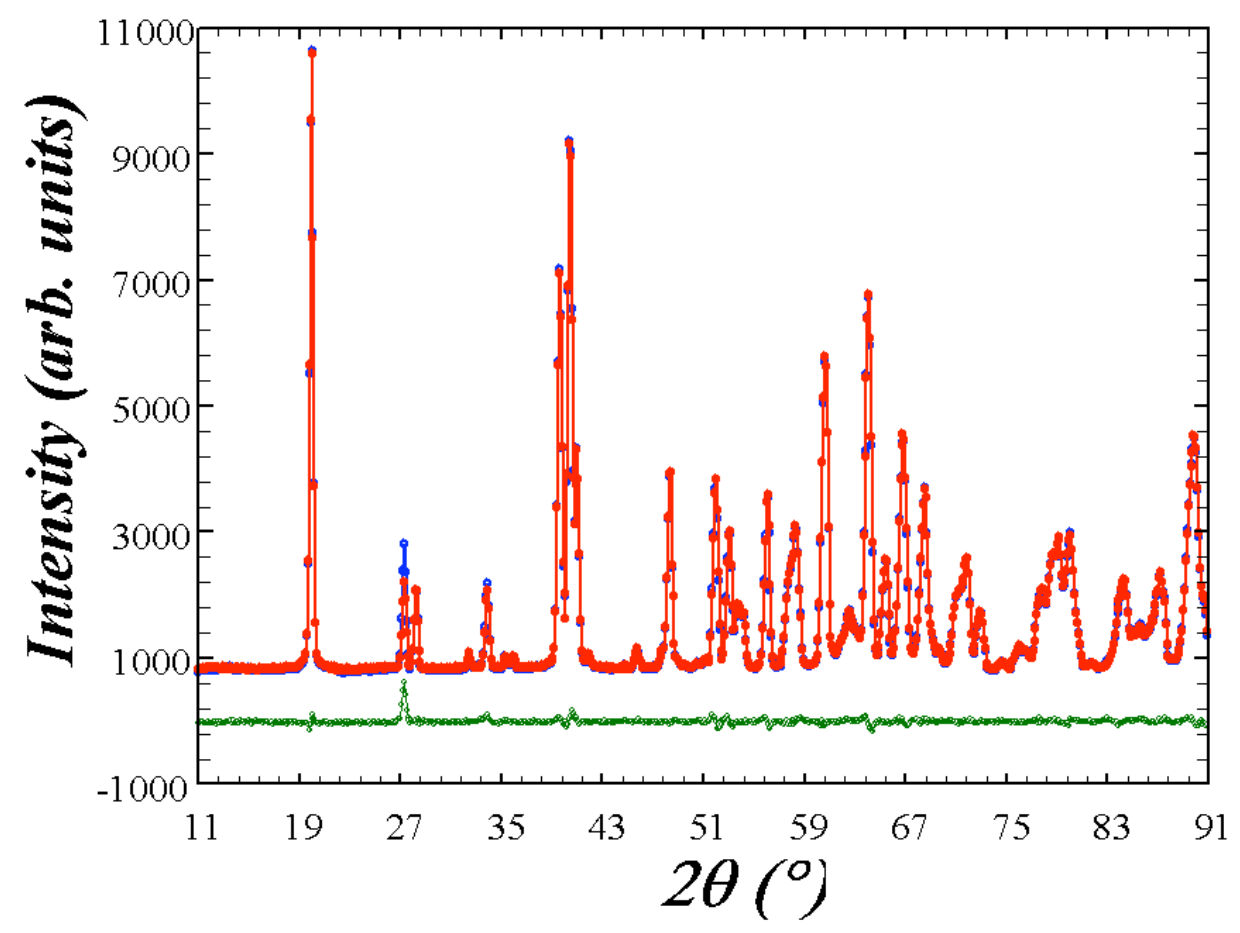


Figure S7. Observed profiles of the neutron powder diffraction pattern of $\mathrm{Cu}_{3}(\mathrm{OD})_{2}\left(\mathrm{MoO}_{4}\right)_{2}$ at $1.5 \mathrm{~K}$ (blue) and $17.8 \mathrm{~K}$ (red) and their difference (green).

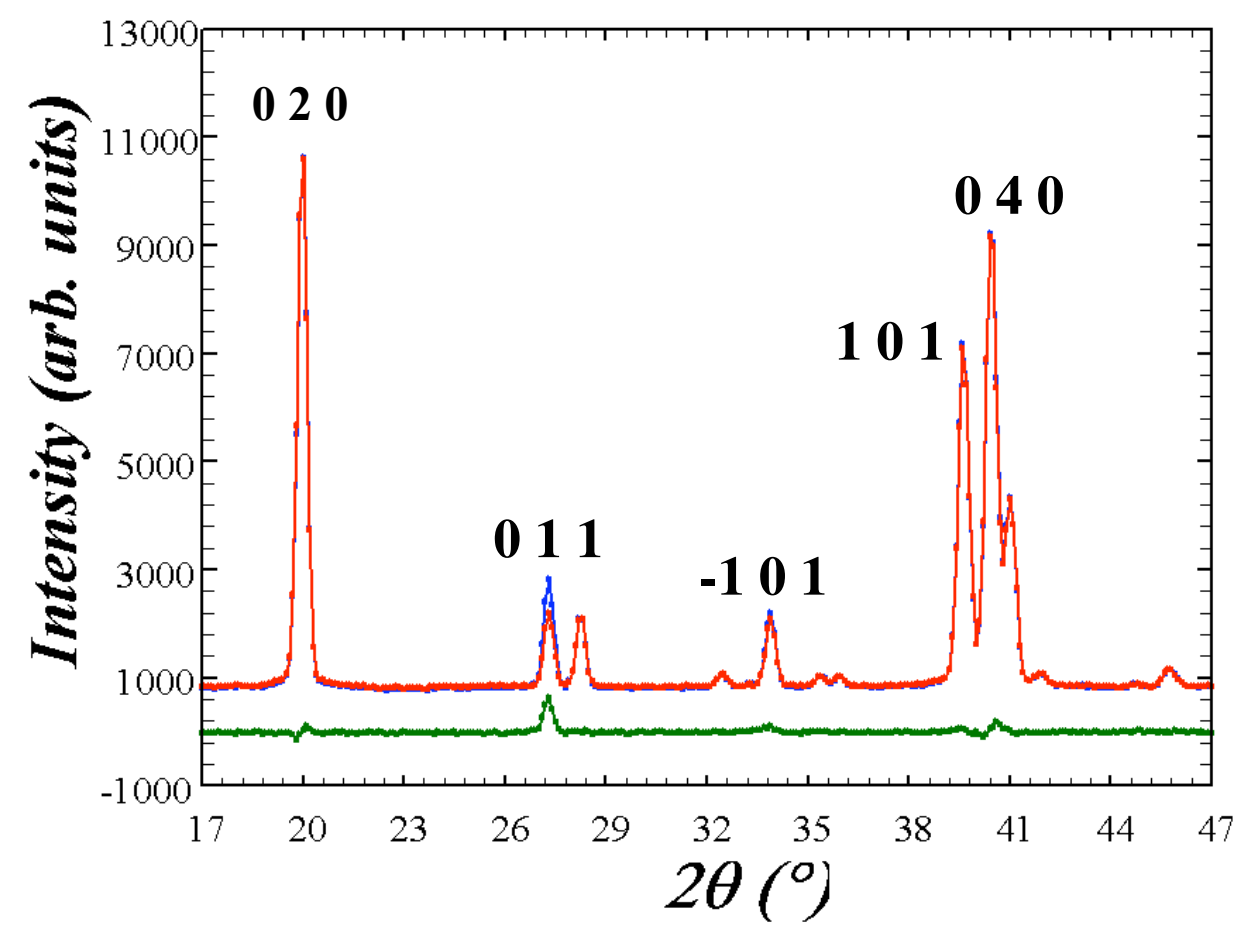

Figure S8. Expanded range of the observed profiles of the neutron powder diffraction pattern of $\mathrm{Cu}_{3}(\mathrm{OD})_{2}\left(\mathrm{MoO}_{4}\right)_{2}$ at $1.5 \mathrm{~K}$ (blue) and $17.8 \mathrm{~K}$ (red) and their difference (green) including the indexes of the magnetic Bragg reflections. 